\section{M39 SCREENING FOR TUBERCULOMAS IN PATIENTS WITH MILIARY TUBERCULOSIS - WHAT MODALITY OF IMAGING SHOULD WE BE USING?}

R Ghani, H Durkan, L John, R Davidson, J Buckley. Department of Tropical Medicine and Infectious Diseases, Northwick Park Hospital, North West London Hospitals NHS Trust, London, UK

\subsection{6/thoraxjnl-2014-206260.427}

Introduction and objectives NICE guidance advises neuroradiology to investigate CNS signs in patients with miliary tuberculosis (TB). The aims of our study were to describe our population of patients with tuberculomas in the presence of miliary disease and identify any clues to the best radiological modality.

Methods The radiology and clinical history was retrospectively reviewed for all patients treated for miliary tuberculosis at one centre between 01/01/2009 and 31/12/2013.

Results 53/1650 (3.2\%) of patients during this period were diagnosed with miliary (disseminated) tuberculosis. 27/53 (50.9\%) underwent further neuroimaging. 10/53 (18.9\%) miliary TB patients had evidence of tuberculomas on neuroimaging (M:F $6: 4$, age range 22:81). 2/10 had evidence of tuberculomas on both CT (2/2 with contrast) and MRI, 5/10 had a negative CT (2/ 5 with contrast) but an MRI result which revealed tuberculomas. 3 patients did not have a CT scan (MRI only). All 10 patients were HIV negative and had fully sensitive TB, 9/10 had neurological signs which warranted the subsequent neuroimaging.

Conclusion Tuberculomas are seen in a fifth of patients with miliary tuberculosis. Based on our findings, guidelines should be adapted to suggest that both use of contrast and MRI should be utilised preferentially.

\section{M40 TACKLING POOR ATTENDANCE TO TUBERCULOSIS CLINIC - WHO, WHY AND WHAT CAN BE DONE}

EK Denneny, SE Black, Y Bogle, VM Macavei, TC O'Shaughnessy, VLC White, H Kunst, NP Jayasekera. Barts Health NHS Trust, London, UK

\subsection{6/thoraxjnl-2014-206260.428}

Introduction Despite efforts to improve Tuberculosis (TB) services, disease rates remain high (UK national average 14.4 per 100,000). We believe one of the ongoing challenges is engaging patients in attending outpatient clinics for care. However, there is no current UK data evaluating poor attendance to TB clinic.

Aim To identify reasons for patient's not attending TB clinic, in order to implement service improvements and increase patient engagement.

Methods We conducted a prospective study reviewing the number of Did Not Attends (DNAs) to our TB clinic over a six-week period (April to June 2014). We evaluated data, usually obtained from patients who are contacted after they DNA, and cross referenced this with the trust electronic database. Data obtained included patient demographics, stage of $\mathrm{TB}$ treatment, route of referral, reasons for non-attendance and accessibility to clinic.

Results 63 of 385 patients (16\% - 42 males, 21 females) did not attend their TB clinic appointments compared to $15 \%$ for non-TB respiratory appointments in this time. 64\% were contactable $(25$ males, 15 females). Median age was 32 (range 17-78 years), which included 16 ethnicities and seven languages. $62.5 \%$ were followup appointments and $37.5 \%$ were new. $27.5 \%$ had TB previously. Stage of TB treatment included: completed (17.5\%), current (25\%), none (57.5\%). Referral route included GP (40\%), hospital $(32.5 \%)$ and contact tracing (27.5\%). 59\% were aware of their appointment but were unable to attend due to other engagements. $41 \%$ stated they had not received a letter informing them of their appointment, $13 \%$ of these patients had relocated to another area and not updated their address. $8 \%$ of patients highlighted problems with transport leading to difficulties accessing the clinic.

Conclusions Communication to inform patients about appointments needs to be improved by both the referring and TB service. Utilising information technology and community links may improve patient education and therefore engagement with services. Experiencing the patient's journey will highlight further areas for development.

\section{M41 RECURRENT TUBERCULOSIS AND ITS RISK FACTORS IN THE UK'S LARGEST TB CENTRE}

K Avery, R Ghani, J Buckley, L John, RN Davidson. Department of Tropical Medicine and Infectious Diseases, Northwick Park Hospital, North West London Hospitals NHS Trust, London, UK

\subsection{6/thoraxjnl-2014-206260.429}

Objective To describe tuberculosis (TB) relapse/recurrence in patients treated at the UK's largest TB centre and identify characteristic which predicted recurrence.

Design Retrospective observational cohort study.

Methods All patients treated at our centre between 1st Jan 2002-31st Dec 2013 were identified from the local TB register. We excluded patients who died due to TB or whose outcome was unknown. Details of patients with more than one notification episode of $\mathrm{TB}$ were obtained from patient records.

Results In total, 3534 patients were treated for TB during the 12 -yr period. After exclusions, 3515 patients were included in the study. Of these, 42 patients had two notifications of TB; none were treated more than twice.

Of these 42, we considered 14 to be true relapses/recurrences. 28 patients were considered on review not to have had a true relapse/recurrence: of these, 11 had their first treatment episode at a different centre; 9 were re-starts of treatment because of non-adherence during the first TB episode; 2 had intracranial tuberculomas diagnosed within 12 months of initial episode; 6 were errors in notification.

Of 14 patients considered to be true relapses/recurrence, 6 were microbiologically confirmed on relapse/recurrence and a further 8 were re-treated on clinical grounds. None exhibited drug resistance and 2 were HIV positive. The 14 true relapse/

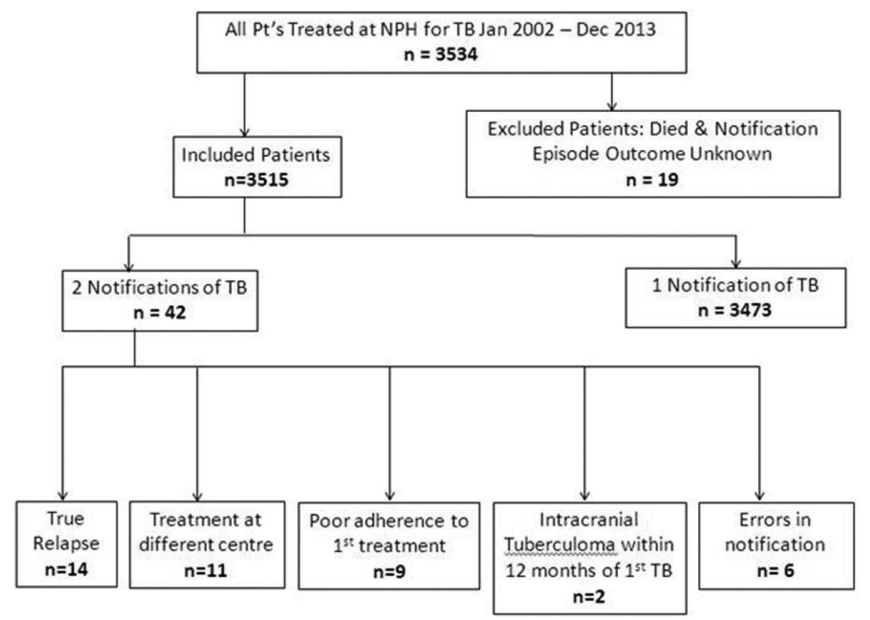

Abstract M41 Figure 1 
recurrence patients had mean age $=42$ yrs (range 18-83 yrs) and 6 were males. The sites of relapse were: pulmonary in 6 cases, 3 patients had intracranial tuberculomas, 2 patients had bony TB, 3 patients had TB lymphadenitis. The mean time to relapse/recurrence was 41 months (range 2-96 mo). All 14 patients responded favourably to re-treatment.

The true relapse rate of TB treated at the centre was $0.4 \%$. The age, gender and ethnicity of the relapse cases were similar to the overall TB case-mix.

COnclusions Our true relapse/recurrence rate of TB is very low, and had no obvious risk factors. We cannot determine retrospectively whether these were recurrence or reinfection, but strain typing (DNA fingerprinting) could differentiate these.

\section{M42 INCREASING COMPLEXITY OF TREATING TB IN OLDER PATIENTS}

J Barrett, GA O'Hara, A Nundoll, N Price, H Milburn, RAM Breen. Guys and St Thomas; NHS Foundation Trust, London, UK

\subsection{6/thoraxjnl-2014-206260.430}

Introduction Older adults remain an important reservoir of tuberculosis (TB) infection in the UK. Waning cellular immune responses, more frequent co-morbidities such as diabetes and malignancy, and increased polypharmacy may all modulate clinical presentation, treatment tolerability and ultimately outcomes when compared to younger individuals with TB. We sought to investigate this in our population.

Methods Retrospective study of all adults over 60 diagnosed with TB during a five year period at one hospital trust. Case-note and electronic record review established baseline disease features, comorbidities, pre-morbid immune suppression including HIV status, TB-related outcomes and death. A randomly selected control group of identical size, containing adults aged 16-59 who were treated for TB during the same period, was used for comparison.

Results Forty-eight cases aged $>60$ years at TB diagnosis were identified. The case and control groups are described in the Table. Multi-lobar pulmonary disease was significantly more common in the $>60$ year old, as was diabetes, other significant co-morbidities and non-HIV immune suppression. Whilst treatment regimen discontinuation or alteration was more common in the $>60$ year old group (7 (14\%) versus $3(6 \%)$ if 60 years old but none $(0 \%)$ of the younger group; whilst deaths after completion of TB treatment have been observed in $8(17 \%)$ and $1(2 \%)$ of cases respectively (no post-treatment death was related to $\mathrm{TB}$ in either group).

\begin{tabular}{llll}
\hline Abstract M42 Table 1 & & \\
\hline & $\begin{array}{l}<0 \text { years old } \\
(\mathbf{n}=\mathbf{4 8})\end{array}$ & $\begin{array}{l}>60 \text { years old } \\
(\mathbf{n}=48)\end{array}$ & \\
\hline Mean age at diagnosis (range) & $34(16-57$ years) & $71(60-88$ years) & \\
Male & $24(54 \%)$ & $33(69 \%)$ & \\
Pulmonary disease & $34(70 \%)$ & $33(69 \%)$ & \\
- multi-lobar & $9(26 \%)$ & $17(52 \%)$ & $\mathrm{p}=0.046$ \\
Culture-confirmed TB & $38(79 \%)$ & $36(75 \%)$ & \\
HIV-infected & $6(13 \%)$ & 0 & $\mathrm{p}=0.026$ \\
Diabetes & 0 & $13(27 \%)$ & $\mathrm{p}<0.01$ \\
Significant immune suppression & $8(17 \%)$ & $29(60 \%)$ & $\mathrm{p}<0.01$ \\
Significant co-morbidities & $14(35 \%)$ & $36(75 \%)$ & $\mathrm{p}<0.01$ \\
\hline
\end{tabular}

Conclusions Our observations of older patients presenting with more extensive pulmonary disease, increased pre-diagnosis immune suppression and co-morbidities, as well as more frequent TB-related deaths and TB-regimen alterations, suggest that this group of TB patients are frequently in need of more intensive support during treatment than their younger counterparts. However if treatment can be tolerated and completed than TB outcomes do not appear to be affected by age.

\section{M43 THE ACCURACY OF CLINICAL TB DIAGNOSES IN CULTURE NEGATIVE PATIENTS}

L Maynard Smith, NP Jayasekera, VM Macavei, TC O'Shaughnessy. Newham University Hospital, London, UK

\subsection{6/thoraxjnl-2014-206260.431}

Background NICE guidelines for TB diagnosis recommend that sputum is obtained for culture for all suspected cases of pulmonary $\mathrm{TB}$, and biopsies for all cases extrapulmonary TB. As results can take 6 weeks, treatment initiation decisions are frequently made without microbiological confirmation.

Aim This study set out to examine the accuracy of clinical diagnoses in a high incidence area, and the basis for these decisions. Methods The data entered onto the national TB database was used to obtain a list of patients for whom no culture results had been recorded. Clinic letters, laboratory records and imaging were examined to determine whether samples had been sent for culture, how diagnoses were made in the event of negative results, and if alternative diagnoses were concluded.

Results Of 323 patients on the database, $7 \%$ had no samples sent for culture. There were 109 culture negative patients, of whom 13 (4\% all cases) had alternative diagnoses. A combination of relevant history and imaging was the most commonly used method of diagnosis when culture was negative (47\%). Histology was used in $17 \%$ patients and Mantoux or IGRA testing supported initiating treatment in $39 \%$ cases. The database was missing positive culture results for 102 patients, of which four were MDR TB.

Conclusions In this study, we found accurate initial clinical diagnoses, with only $4 \%$ patients subsequently obtaining alternative diagnoses. Most diagnoses were made on the basis of relevant history and imaging. Of concern are the $7 \%$ patients for whom tissue was never sent for culture. This is likely to be an underestimate when including all patients initially suspected of TB, raising the possibility of missed diagnoses. The utility of Mantoux and IGRA testing in active disease is now disputed. It is hoped with inter-specialty education regarding the importance of culture and futility of immunological based assays, the proportion of patients with suspected TB who have sputum or tissue sent for culture increases. Accurate recording of MDR-TB on the national TB database needs to be improved, to enable efficient monitoring of intervention programmes.

\section{COPD: co-morbidities, deficiencies and interventions}

\section{M137 CAN STEROID INSENSITIVITY IN COPD PATIENTS BE RESTORED USING VITAMIN D?}

D Mukherjee, D Parekh, R Dancer, M Ungurs, H Khiroya, AM Turner. Queen Elizabeth Hospital Research Laboratories, University of Birmingham, Birmingham, UK

10.1136/thoraxjnl-2014-206260.432 